\title{
Single-vehicle data of highway traffic: microscopic description of traffic phases
}

\author{
Wolfgang Knospe ${ }^{1}$, Ludger Santen ${ }^{2}$, Andreas Schadschneider ${ }^{3}$ and Michael Schreckenberg ${ }^{1}$ \\ 1 Theoretische Physik, Fakultät 4, Gerhard-Mercator-Universität Duisburg, Lotharstr. 1, 47048 Duisburg, Germany \\ ${ }^{2}$ Fachrichtung Theoretische Physik, Universität des Saarlandes, Postfach 151150, 66041 Saarbrücken, Germany \\ ${ }^{3}$ Institut für Theoretische Physik, Universität zu Köln, Zülpicher Str. 77, 50937 Köln, Germany
}

(October 24, 2018)

\begin{abstract}
We present a detailed analysis of single-vehicle data which sheds some light on the microscopic interaction of the vehicles. Besides the analysis of free flow and synchronized traffic the data sets especially provide information about wide jams which persist for a long time. The data have been collected at a location far away from ramps and in the absence of speed limits which allows a comparison with idealized traffic simulations. We also resolve some open questions concerning the time-headway distribution.
\end{abstract}

\section{INTRODUCTION}

Like in every other field of physics the modeling of traffic flow is based on the analysis of empirical data. In the last few years, several empirical studies were carried out in order to obtain a more profound picture of highway traffic. Many modeling approaches have been suggested which try to capture the essential parts of the human driving behavior (see [1,2] for an overview). On the one hand, the empirical observation of highway traffic helps to identify and to characterize traffic phases and the transitions between them. On the other hand, the theoretical modeling allows simulations of large traffic networks and opens perspectives for future applications like traffic forecasts and dynamic route guidance systems. However, realistic model approaches of highway traffic have to be based on empirical results and especially the microscopic modeling of traffic requires information on the driving behavior of the vehicles in the various traffic states.

Much progress has been made in the last few years in analyzing empirical data. Moreover, the technical development of the measurement techniques and the improved equipment of roads with detectors allows to observe spatio-temporal structures [3] and even to analyze single-vehicle data [4. 5]. The analysis of large sets of minute averaged traffic data has revealed the existence of three traffic phases, namely (i) free flow, (ii) synchronized traffic and (iii) wide moving jams. This three state picture is nowadays widely accepted, although other interpretations of empirical data have been made 68 .

Free flow traffic can be characterized by a large average velocity while congested traffic is simply the opposite of free flow. An important property of congested traffic is that the average velocity of the different lanes can be synchronized [9, 10] leading to large correlations between the velocity measurements on neighboring lanes. Congested traffic can be divided into synchronized traffic and wide moving jams.

In synchronized traffic, which is mainly observed at on- and off-ramps, the velocity is considerably smaller than in free flow but the flow can still have large values.
Moreover, the large variance in density and flow measurements cannot be described by a functional relationship which becomes visible in an irregular pattern in the fundamental diagram. Therefore, the cross-correlation between flow and density vanishes and thus allows to identify the synchronized state [4. In 11 the following picture of the synchronized state and a possible jam formation was developed: While the downstream front of the synchronized traffic region is usually fixed at an onor off-ramp the upstream front is separated from free flow by the so-called pinch region. In the pinch region narrow jams are formed. In contrast to wide jams, narrow jams consist of only two fronts where the vehicle speed and the density sharply changes whereas the width of the fronts of wide jams is negligible compared to the bulk region between them. These jams can merge and form wide jams.

Wide (moving) jams are regions with a very high density and negligible average velocity and flow. The width of these structures is much larger than its fronts at the upstream and downstream ends where the speed of vehicles changes sharply. A wide jam can be characterized by a few parameters, e.g., the velocity with which it moves upstream. The velocity of the downstream front and the corresponding flow rate is only determined by the density inside a wide jam and the delay-time between two vehicles leaving the jam [12]. In contrast to synchronized traffic wide jams can propagate undisturbed through either free flow and synchronized traffic without impact on these states which thus allows their coexistence.

In order to identify the different phases it would be ideal to have data from a series of detectors in order to identify the traffic states by their spatio-temporal properties [3]. Since the data set used in this study is recorded at a single measurement location we use autocorrelation and cross-correlation functions to determine these properties locally.

The analysis of single-vehicle data gives important information about the driving behavior of the vehicles in the various traffic states. Unfortunately, up to now only a few empirical results of single-vehicle data exist [4, 战] 
which do not provide a consistent picture of the vehicle dynamics. It was shown that the distribution of the time-headways and the velocity-distance relationship do not depend on the density but on the traffic state. In [A] a peak at $1.8 \mathrm{~s}$ was found in the time-headway distribution which was explained with the drivers effort for safety. However, the existence of this peak could not be confirmed in $[5]$. Moreover, in the free flow state platoons of vehicles driving bumper-to-bumper with a time-headway well below $1 \mathrm{~s}$ occur.

The velocity-distance relationship gives information on the adjustment of the velocity on the headway. While in free flow the velocity shows large values even for small headways, in the congested state drivers tend to move slower than the distance allows. Thus, in the free flow regime the movement of the vehicles is uncorrelated although small platoons exist while in the synchronized regime large correlations of the velocity between the vehicles can be found but the distance between them can vary considerably.

In this empirical analysis we try to improve the microscopic understanding of the human driving behavior which helps to validate the properties of models for traffic flow on a qualitative level. It is worth pointing out that the data set underlying our study is one of the largest used so far. In contrast to older studies the data have been collected on a 3-lane road without speed limit and relatively (several kilometers) far away from ramps. The quality of the empirical data enables a detailed insight into the dynamics of the vehicles in wide jams. The location of the detectors far away from ramps and the absence of a speed limit allow a much better comparison with data from idealized traffic simulations without bottlenecks. In analogy to onramps [13], defects introduced by, e.g., the application of a speed limit, considerably affect traffic flow and can trigger distinct traffic states. However, it has been shown recently for a large class of driven diffusive systems (see e.g. [14, 15]) that bottlenecks only select rather than generate the states. This is a very general property of driven system and is well-established in nonequilibrium statistical physics (see ref. [16] and references therein). Furthermore it has also been verified for a large class of traffic models. Therefore our analysis has, compared to measurements at bottlenecks, the advantage that we can observe and analyze pure bulk states. The disadvantage is that congested traffic is less often observed, which implies that there is need for quite long observation periods. However, the data set, which is analyzed throughout this article, includes all three types of highway traffic. Our results are therefore of direct relevance for the modeling of traffic flow on highways.

\section{EMPIRICAL DATA}

The data sets were collected at different locations on two highways in North-Rhine-Westfalia, Germany. The first set is provided by two detectors, one for each driving direction, on the A3 between the junction DuisburgWedau and the highway-intersection Kreuz-Breitscheid (see Fig. 11). A detector consists of three inductive loops, one for each lane. The location of the detector is about $5.5 \mathrm{~km}$ from the highway intersection Breitscheid and about $2.2 \mathrm{~km}$ from the junction Duisburg-Wedau. It is important to note that there is no speed limit. The data set was measured between 03-30-2000 and 05-16-2000 and comprises 48 days. The second and the third data set was measured by four detectors, one for each driving direction consisting of three inductive loops, at two locations on the A1 between the highway intersection Leverkusen and the junction Burscheid (see Fig. 1). Note, that for storage reasons, in the third data set only the vehicles on the right and the middle lane were detected. The detectors are located $6.150 \mathrm{~km}$, respectively $7.300 \mathrm{~km}$ from Leverkusen and $3.950 \mathrm{~km}$, respectively $2.800 \mathrm{~km}$ from Burscheid with a distance of $1.150 \mathrm{~km}$ between the two measurement locations. The data were collected during two periods (08-02-2000 to 08-20-2000 and 02-01-2001 to 02-27-2001) which comprises a total measurement time of 46 days. Note, that the highway has three lanes and there is no speed limit. In total, on 94 days about 9.5 million cars were measured.

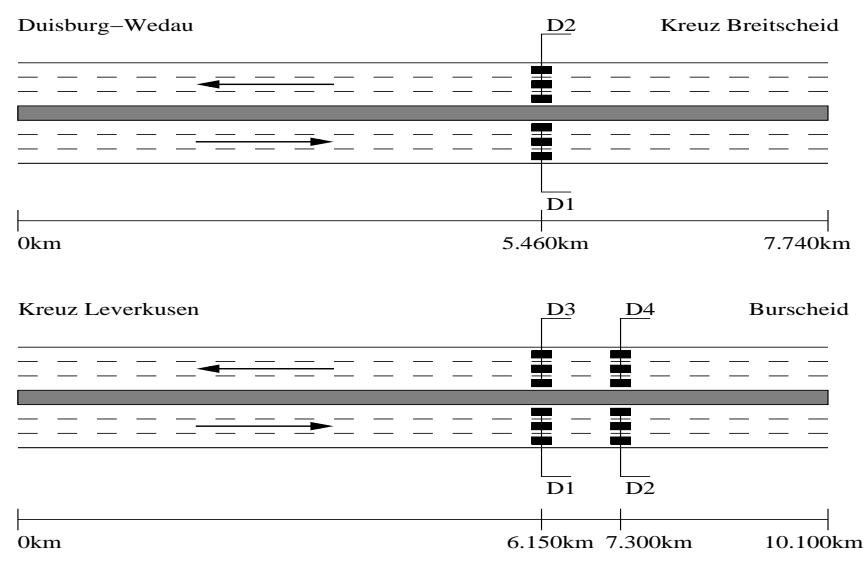

FIG. 1. Location of the detectors of the first data (top) set measured on the A3 near Duisburg and of the second and third data set (bottom) measured on the A1 near Leverkusen.

All of the inductive loops were able to measure singlevehicle data. A data set of one vehicle comprises the time (in hundredth seconds) at which the vehicle reaches the detector, the lane the car is detected, the type of the vehicle (car, truck), the velocity in $\mathrm{km} / \mathrm{h}$ and its length in $\mathrm{cm}$. The length of a vehicle is calculated with an accuracy of 1 $\mathrm{cm}$. The lower bound for the velocity measurement is 10 
$\mathrm{km} / \mathrm{h}$, i.e., velocities of slower vehicles are not measured. Then, instead of the length of the vehicle, the time the inductive loop is covered is given in hundredth seconds. Note, that the velocity is theoretically calculated internally via the time $\Delta t$ a vehicle needs to pass a detector of a given length with an accuracy of $1 \mathrm{~km} / \mathrm{h}$. However, since $\Delta t$ is only measured in milliseconds and no floating points for the calculation of the velocity are used, it is possible that a velocity may correspond to more than one $\Delta t$ and/or a difference of one millisecond in $\Delta t$ leads to a velocity difference larger than $1 \mathrm{~km} / \mathrm{h}$. As a result, small differences in $\Delta t$ cannot be resolved while some velocities cannot be measured.

The third data set includes the electric signal inducted in the loops when a car passes the detector. These data comprise the time (in milliseconds) the vehicle arrives at the detector and the time-series of the height of the electric signal that is sampled at 125 points in one second.

In addition to the single-vehicle data of the first and the second data set, one minute averages of the traffic data are collected. These data comprise the number of cars and the number of trucks, the mean velocity of cars, the mean velocity of trucks and the mean velocity of all vehicles. Occupancy (occ) rather than density is used as an estimation of the density because of the ease of measuring and its independence from the vehicle lengths. It is simply given by the fraction of time the detector is covered by a vehicle.

The spatial density can be calculated from the occupancy. An occupancy occ $=1$ means that the vehicles are driving bumper-to-bumper which leads to a spatial density $\rho_{\max }$ of

$$
\rho_{\max }=\frac{N(T)}{\sum_{t \leq t_{n}<t+T} l_{n}}=\frac{1}{L}
$$

with the mean length $L$ of the vehicles and the number $N(T)$ of the vehicles passing the detector in time $T$. In this study the value of $T$ is set to $60 \mathrm{~s}$. $l_{n}$ is the length of the $n$-th vehicle measured at time $t_{n}$. For the spatial density $\rho_{s}$ estimated by occupancy

$$
\rho_{s}=o c c \cdot \rho_{\max }=o c c \cdot \frac{N(T)}{\sum_{t \leq t_{n}<t+T} l_{n}}=\frac{o c c}{L}
$$

holds 7 Throughout this paper the terminology "density" is used for occupancy if not stated otherwise and the occupancy is given in percent.

Since large occupancies (e.g., a vehicle blocks the detector for more than one minute) are not distributed on

\footnotetext{
${ }^{*}$ The calculation of the spatial density by means of local measurements is only valid under the assumption of a constant velocity of the vehicles during the averaging process.
}

minute intervals by the counting device but the occupancy is just related to the interval the vehicle is measured, a cut-off of the minute averaged data at $100 \%$ was introduced. Thus, the explicit calculation of the occupancy of the time interval $[t, t+T]$ by means of the single-vehicle data via

$$
o c c(t)=\frac{1}{T} \sum_{t \leq t_{n}<t+T} \Delta t_{n}=\frac{1}{T} \sum_{t \leq t_{n}<t+T} \frac{l_{n}}{v_{n}}
$$

with the time $\Delta t_{n}$ the detector is occupied by car $n$ of length $l_{n}$ and velocity $v_{n}$ allows to determine the occupancy more accurately compared to the one minute data because occupancy overlaps from minute to minute can be considered. The minute data therefore are only used to verify the averaged single-vehicle data.

Furthermore, occupancy can explicitly be related to the various traffic states. In contrast, the hydrodynamical relation

$$
\rho=\frac{J}{v}
$$

where $J$ is the flow (which is proportional to the number $N$ of vehicles passing the detector in a time interval $\Delta t$ ) and $v=\sum v_{n}(t) / N$ is the average velocity of the vehicles tends to underestimate the density since the velocity of moving cars is detected (see [4]). This leads especially in the congested state to very small densities while in free flow traffic both methods give the same results.

The time resolution of the single-vehicle data allows the calculation of the time-headway $t_{h}$ and the distanceheadway gap of the $n$-th vehicle via

$$
t_{h}(n)=t_{n}-t_{n-1}-\frac{l_{n-1}}{v_{n-1}}
$$

and

$$
\operatorname{gap}(n)=v_{n}\left(t_{n}-t_{n-1}\right)-l_{n-1}
$$

under the assumption if of a constant $v_{n}$ and $v_{n-1} \cdot t_{n}$ denotes the time the $n$-th vehicle passes the detector, $l_{n}$ and $v_{n}$ its length and velocity. The passing time are measured by the internal clock with an accuracy of 0.001 s. In the output these signals are provided in units of hundredth seconds. The achievable resolution of spatial measurements is $1 \mathrm{~cm}$. These values are of course lower

\footnotetext{
${ }^{\dagger}$ The usage of the velocity $v_{n}$ is somehow arbitrary since $v_{n-1}$ can also be taken which leads to the calculation of the headway in upstream direction rather than downstream. However, the difference between both results is negligible because on small distances the surroundings upstream and downstream of the measurement location should be homogeneous.
} 
bounds for the real error bars. In particular for the distance headways we expect much uncertainties, roughly of the order of $\sim 1 \mathrm{~m}$. Unfortunately, it is difficult to calculate precisely one realistic errorbar, because they depend on, e.g., the velocity of the cars and the actual value of the distance.

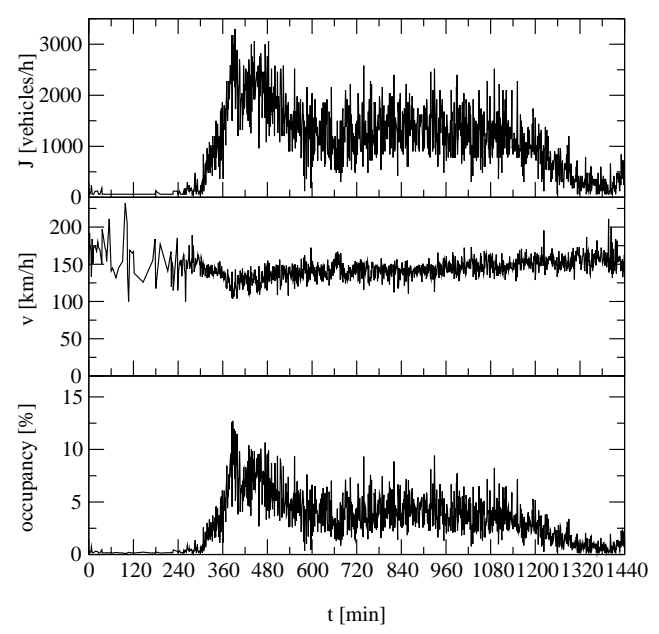

FIG. 2. Time-series in the free flow regime of the flow, the velocity and the occupancy on the left lane at detector D1 on the A3 on the $04-28-2000$.

In order to allow the calculation of spatio-temporal correlations on the A1, the two measurement locations D3/D4 and D2/D4 are synchronized by means of radio controlled clocks.

\section{FREE FLOW}

Free flow is the traffic state most frequently observed in the data set and is characterized by a large flow $J$ and velocity $v$ and a small density (Fig. 2). While daily variations of the flow and the density become visible in the time-series , the velocity remains nearly constant over the whole day.

\footnotetext{
${ }^{\ddagger}$ Quantitatively they can be seen in the behavior of the autocorrelation functions [4].
}

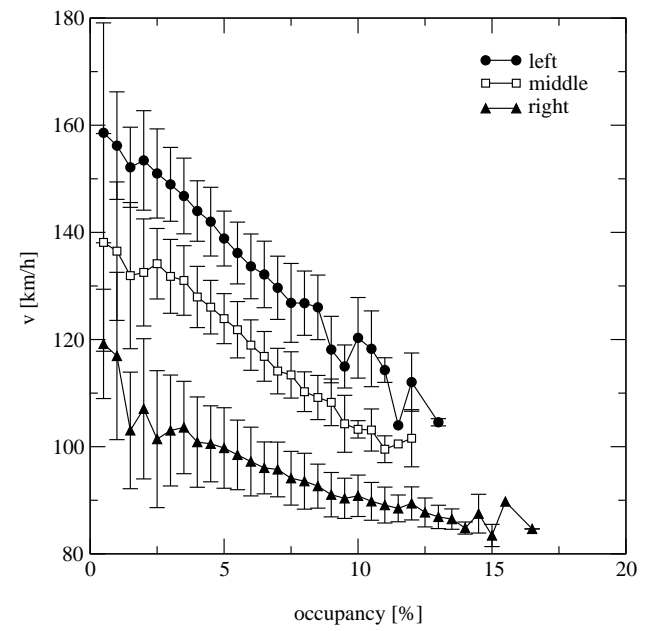

FIG. 3. Average velocity dependent on the density for the three lanes at detector D1 on the A3 on the 04-28-2000.

Due to legal restrictions in Germany vehicles have to drive on the right lane and the left and the middle lane should be used only for overtake maneuvers. Thus, the lanes segregate into fast and slow lanes which results in a negative velocity gradient from left to right (Fig. 3). This effect becomes more pronounced in the absence of a speed limit. As a consequence, the flow and the velocity decreases from the left to the right lane, whereas the density increases.

Next we analyze the velocity distributions in free flow traffic (Fig. 1). The velocity calculated by the detector is simply the length of the detector divided by the time $\Delta t$ the vehicle takes to pass the detector, that is measured directly. In our case $\Delta t$ is measured with a precision of milliseconds, but the corresponding velocity is given in units of $\mathrm{km} / \mathrm{h}$ since only integer arithmetics was applied internally. This way of calculating the velocity does not use the full precision of the measurement, but even worse, it leads to an uneven sampling of the velocities. The latter point can be avoided by recovering the corresponding traveling time $\Delta t$ from the velocity given in the data set. For large velocities $(v>110 \mathrm{~km} / \mathrm{h})$ at one hand we obtain a unique value of $\Delta t$. For smaller velocities on the other hand each velocity corresponds to a range of possible traveling times. If the same velocity results for $k$ different traveling times, we simply increase the statistical weight of each possible value of $\Delta t$ by $1 / k$. This procedure leads to smooth traveling time distributions. If one is interested in the velocity distributions one is still left with a problem, i.e. the velocity intervals are that correspond to a given traveling time are not of equal length. Therefore we calculated the velocity, that corresponds to a certain travelling time, with a high numeri- 
cal resolution, i.e. $10^{-2} \mathrm{~km} / \mathrm{h}$, and interpolated linearly between the two measurements. In order to obtain a normalized distribution the interpolated values have to be devided by the length of the interval. In the final figure, however, we use a resolution of $1 \mathrm{~km} / \mathrm{h}$, by averaging over the evenly sampled and highly resolved velocity distribution. This procedure leads obviously to very smooth velocity distributions.

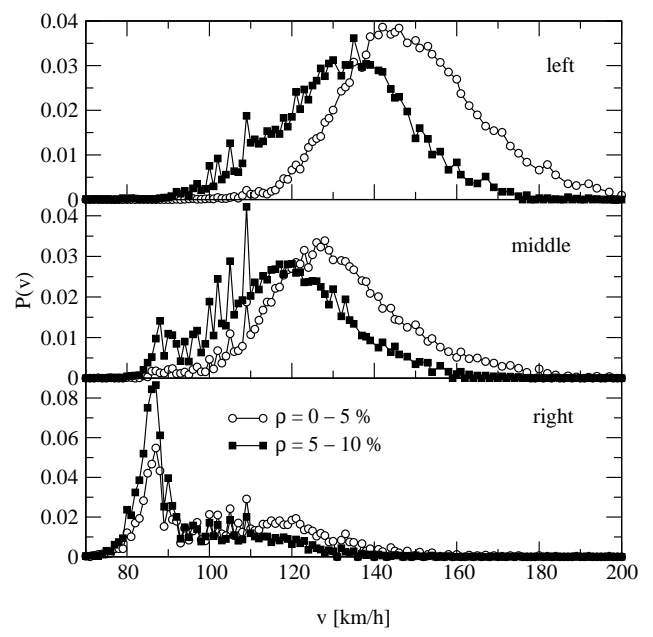

FIG. 4. Distribution of the velocity on the three lanes at detector D1 on the A3 on the 04-28-2000.

On the left lane the velocity distributions can be nicely fitted to a Gaussian distribution, for densities between $5-10 \%$. This is in agreement with earlier investigations, also finding Gaussian distributed velocities, for a low portion of trucks [17]. For lower densities $(0-5 \%)$, however, we observe a slight asymmetry of the distribution function. In order to quantify this asymmetry we divided the distribution function into two parts, one containing all velocities below the maximum of the distribution, and the other for larger velocities. Both parts are in agreement with different Gaussian distributions (Fig. D).

The form of the distribution function on the middle and right lane is different, because they can be used by trucks. The trucks lead to a second maximum of the velocity distribution. For these two lanes the velocity distribution can be viewed as a superposition of a "car" and a "truck" velocity distribution, both of Gaussian form in the density interval $5-10 \%$. For lower densities we find again an asymmetry of the distribution function that

\footnotetext{
${ }^{\S}$ The final distribution function does not change if a higher resolution is chosen.
}

is even more pronounced than for the left lane. For an overview, the fit-parameters are summarized in table 1 .

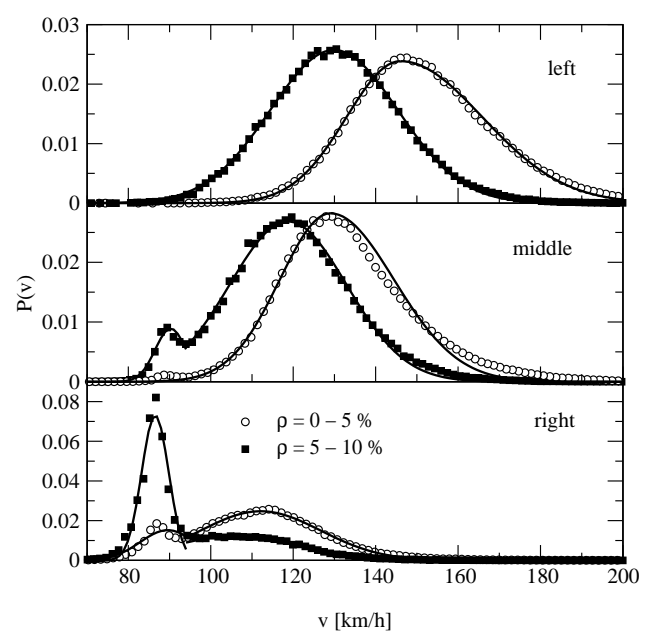

FIG. 5. Distribution of the recalculated velocity (see text). The solid lines represents best fits with distributions of Gaussian type. For distribution functions that have two maxima a superposition of two gaussian distributions has been used. For single peaked distributions a simple or, in case of asymmetric distributions, a distribution consisting of two piecewise defined Gaussians has been used as a fit function. (see table $\mathbb{1}$ ).

In the presence of a speed limit it was found that the negligible vehicle interactions are one of the main characteristics of free flow traffic [4]. In contrast, the velocitydensity relationship in Fig. 6 clearly deviates from a constant value. This fact can be explained by the absence of a speed limit.

\begin{tabular}{lllll}
\hline \hline density & distribution & $\mathrm{a}$ & $\mathrm{b}$ & $\mathrm{c}$ \\
\hline \multirow{3}{*}{$0-5 \%$} & left, $v<146 \mathrm{~km} / \mathrm{h}$ & 146.0 & 340.79 & 41.93 \\
& left, $v \geq 146 \mathrm{~km} / \mathrm{h}$ & 146.0 & 789.86 & 41.93 \\
& middle, $v<129 \mathrm{~km} / \mathrm{h}$ & 129.0 & 300.21 & 35.46 \\
& middle, $v \geq 129 \mathrm{~km} / \mathrm{h}$ & 129.0 & 477.137 & 35.46 \\
& right, cars & 112.10 & 424.22 & 40.40 \\
& right, trucks & 89.55 & 88.44 & 65.70 \\
\hline $5-10 \%$ & left & 129.9 & 477.84 & 38.92 \\
& middle, cars & 118.75 & 401.50 & 37.27 \\
& middle, trucks & 90.27 & 28.10 & 112.27 \\
& right, cars & 106.24 & 466.06 & 80.95 \\
& right, trucks & 86.51 & 21.72 & 13.69
\end{tabular}

TABLE I. Fit parameters of the velocity distribution in free flow. As fit function the Gaussian $p(v)=\exp \left(-(v-a)^{2} / b\right) / c$ was used. 
Slow vehicles are responsible for the decrement of the mean velocity with increasing density. The average velocity decreases linearly with the occupancy $(v \approx 160.206 \pm$ $0.4884-(4.44036 \pm 0.1169)$ occ $)$ and consequently the flow quadratically, $J \approx 5.02139 \pm 4.334+(368.543 \pm 2.177)$ occ$(9.54743 \pm 0.2377)$ occ $^{2}$.

In contrast, queuing theory predicts for a M/G/2 queuing system that has to be applied for a three-lane highway [18] a quadratic relationship between density and velocity and a cubic relationship between density and flow, respectively. For two-lane highways (a M/G/1 queuing system) the velocity is expected to depend linearly on the density.

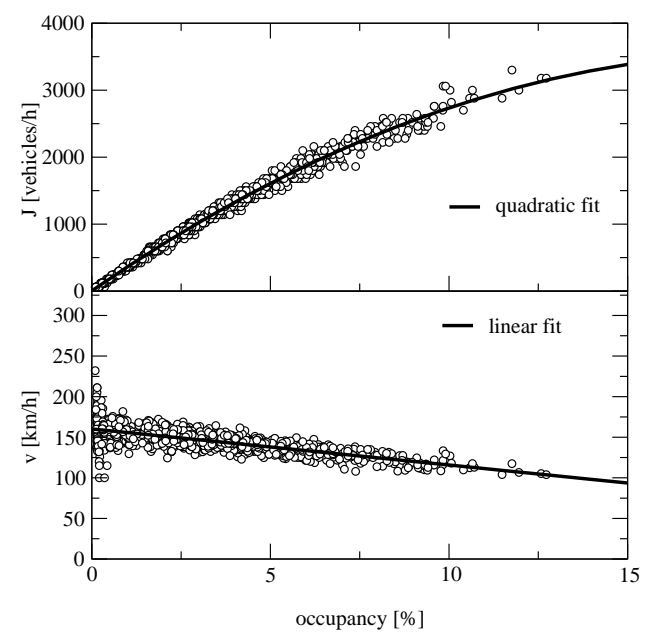

FIG. 6. Fundamental diagram of the left lane for the flow and the velocity in the free flow regime on the 04-28-2000 at detector D1 on the A3. The solid curve gives the best fit for the data.

The discrepancy between queuing theory and empirical data may indicate that either the theory is not appropriate or that the three lanes are not homogeneously coupled. The latter point of view is supported by a correlation analysis of the different lanes, showing that the coupling between left and middle lane is much stronger than between the right and middle lane. It would be interesting to parameterize the partial decoupling of the right lane and to study the modified queuing theory.

The velocity-headway curve illustrates the vehicle's velocity adjustment on the distance-headway. Since cars can move freely, the velocity saturates even for small distances. However, with increasing vehicle interaction the asymptotic value of the velocity decreases (Fig. 7).

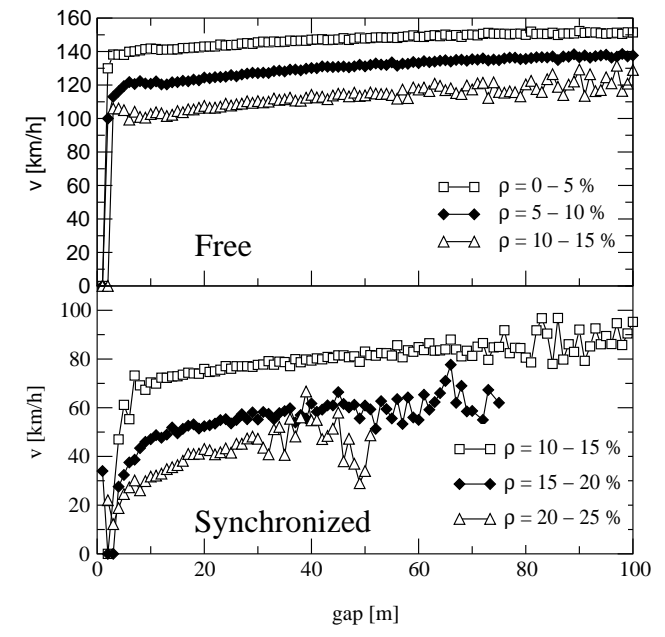

FIG. 7. The mean velocity chosen for a certain distance-headway at detector D1 on the left lane of the A3 for all days with free flow and synchronized traffic.

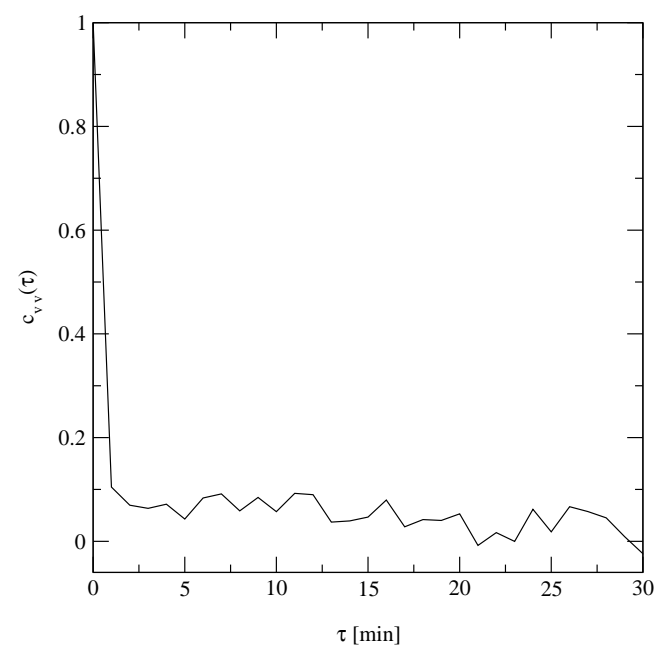

FIG. 8. Autocorrelation of the minute averaged velocity of the left lane on the $04-28-2000$ at detector D1 on the A3.

The vehicle interactions, although visible in the fundamental diagram, are very small. In Fig. \& the autocorrelation (see appendix A) of the one-minute averages of the velocity is shown. In order to eliminate the daily variations the time-series of the velocity was detrended ${ }^{* *}$ by a linear fit. Obviously, the minute averaged veloc-

\footnotetext{
${ }^{* *}$ The data were fitted using a linear regression. This trend was then subtracted from the data points.
} 
ity shows no correlations on time scales larger than one minute since the vehicles are in general driving independently.

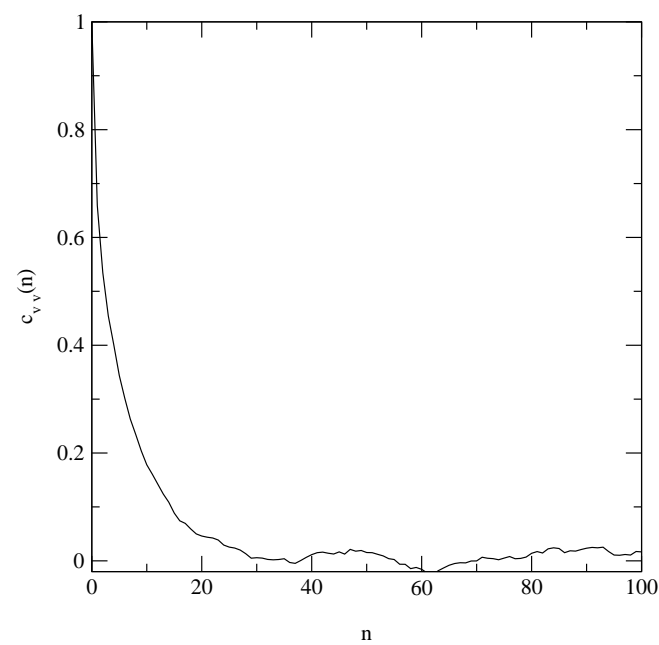

FIG. 9. Autocorrelation of the single-vehicle velocity in free flow of the left lane at detector D1 on the $04-28-2000$ on the A3.

Nevertheless, a closer look on the autocorrelation of the velocity of the single-vehicle data (Fig. 9) reveals correlations on very short time-scales. Like the minute-averaged data, the single-vehicle data were detrended to eliminate daily variations. Now, the autocorrelation function is not time-dependent but depends on the number of vehicles. The strong correlations on short time-scales can be attributed to small platoons of cars driving with nearly the same speed, but with very small headways 此. The platoons are also responsible for small time-headways in the time-headway distribution (Fig. 10). As one can see from Fig. 10 the time-headway distribution differs from

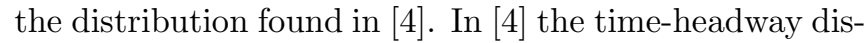
tribution of the single-vehicle data shows the existence of a peak at $1.8 \mathrm{~s}$ for all densities and all traffic states which was explained to be the optimal safe time-headway between vehicles.

This peak was missing in related empirical investigations [5] including the present. In order to clarify the origin of this peak we analyzed the data used in [1] again. A detailed analysis of this data set revealed even more than one peak, but also smaller peaks for other multiples of $0.9 \mathrm{~s}$. Therefore one is let to the believe that these peaks could be artificially generated by a software error of the detection device. Indeed it turned out that in case of a busy detector the data are not directly transfered but stored in a buffer for a time of $0.9 \mathrm{~s}$.

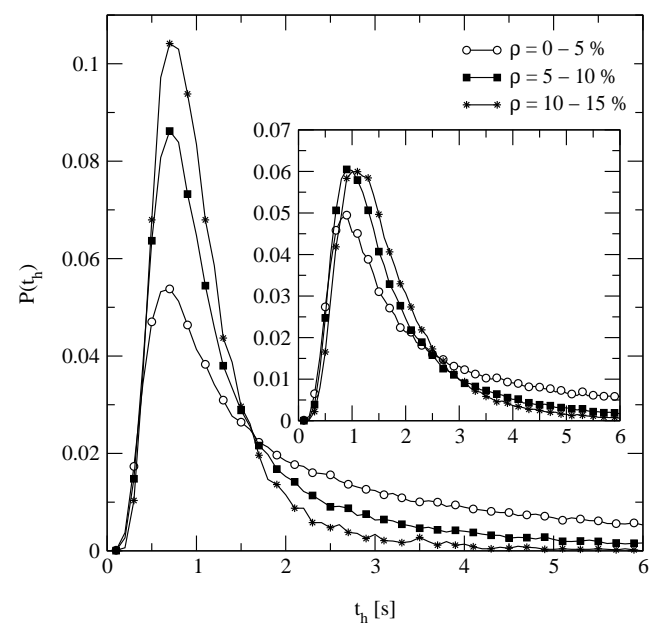

FIG. 10. Distribution of the time-headways of the left lane at detector $\mathrm{D} 1$ on the $\mathrm{A} 3$ for all days of the data set with free flow. The inset shows the time-headway distribution of new measurements on the A1 near Köln-Lövenich.

Since the single-vehicle data contain no time-stamp and the storage time of the internal clock of the computer was used, to each data point in the buffer the same time of measurement is assigned. In the time-headway distribution then the multiples of the buffer size become visible. This is confirmed by new measurements (see inset of Fig. 10) at the detectors D2 and D3 on the A1 near Köln-Lövenich that have also been used in 酒. This data set (like the data sets used in this paper) records the time a vehicle arrives at the detector in hundredth seconds which therefore allows the calculation of the exact time-headway. The new data do not show a peak at $1.8 \mathrm{~s}$ in the time-headway distribution (about 1.5 million cars were detected in 19 days).

At this point we stress the fact that the velocity and length measurements of the single-vehicle data of [- 4 are not affected by the software error and more important, the order of the data is not mixed up. In particular, results of the fundamental diagram, the time-series analysis and the optimal-velocity curve can be reproduced by the data sets used here.

The time-headway distribution found here, shows a strong dependence on the density. However, although the width of the distribution decreases the short time behavior remains nearly unchanged. Not only the maximum of the distribution is at about $0.68 \mathrm{~s}$, but also the values of the shortest time-headways do not change significantly. Note, that the distribution was calculated by considering all days of the data set with free flow traffic. 


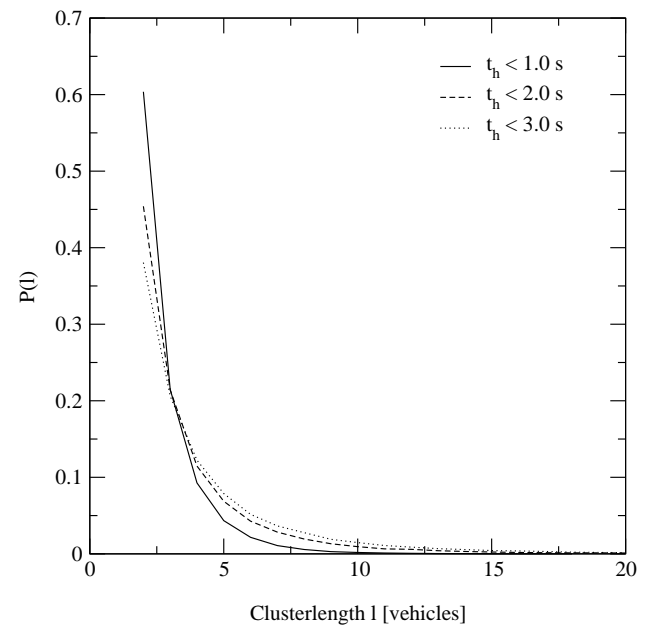

FIG. 11. Cluster length distribution on the left lane in the free flow regime for different time-headways.

If one compares the time-headway distributions of the highway with and without speed limit, one observes that the maximum of the distribution is shifted towards larger time-headways in case of an applied speed limit. This observation shows the importance of anticipation effects in free flow traffic. However, the anticipation of the predecessor's movement leads to serious problems concerning safe driving, because the drivers adjust the distance to the leading vehicle discarding their own velocity.

Another important point is the cut-off of the distribution at small time-headways. In both cases we found that a non-negligible portion of vehicles drives with a temporal distance of the order $\sim 0.2 \mathrm{~s}$, that is comparable to the reaction time of the drivers. These drivers take a very high risk to provoke an accident and would definitively be unable to avoid a crash in case of an sudden obstacle.

It is now possible to estimate the temporal extension of the platoons. From the decay of the autocorrelation of the single-vehicle velocity a platoon length of the order of magnitude of about 10 vehicles follows. Since the timeheadways within a platoon are smaller than the maximum of the distribution (about $1 \mathrm{~s}$ ) this leads to values less than one minute. Thus, these small vehicle clusters cannot be visible in the minute averaged data.

The analysis of the single-vehicle data allows to measure cluster lengths directly. A platoon of length $l$ consists of $l$ consecutive vehicles each having a time-headway smaller than a defined value $\left.\right|^{\text {Tit }}$. By counting the number of succeeding vehicles with time-headways well below a

\footnotetext{
${ }^{\dagger \dagger}$ This value should be of the order of the maximum of the time-headway distribution, i.e., about $1 \mathrm{~s}$.
}

certain value one obtains the cluster length distribution (see Fig. 11 for the distribution of the left lane).

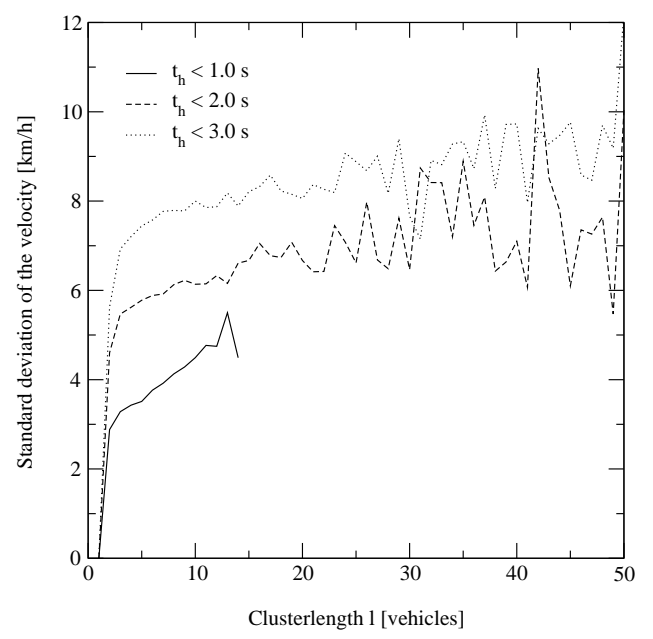

FIG. 12. Average standard deviation of the mean velocity of the vehicles in a cluster of length $l$ on the left lane.

The length of the platoons increases with increasing time-headway, but even with very small time-headways lengths up to 14 vehicles can be measured. In contrast to vehicles on the middle and the right lane, cars on the left lane have no possibility to overtake a slower one. As the length of the platoons decreases from the left to the right lane one can conclude that platoons are simply formed by fast cars piling up behind a slow car (see [19] for a functional relationship of the bunch size distribution).

Driving bumper-to-bumper with large velocity requires an increased attention to velocity fluctuations of the preceding vehicle. As a consequence, the velocity of vehicles inside a platoon is almost synchronized which leads to a slow decay of the autocorrelation function. Driving in a synchronized manner also decreases the velocity differences within the platoon. In Fig. 12 the standard deviation of the velocity of all platoon vehicles is depicted. As one can see, the smaller the time-headway the smaller the velocity differences. Furthermore, the standard deviation saturates very fast so that even large clusters move as synchronized as small ones.

In order to evaluate the stability of the platoons, i.e., identify stable moving structures, we calculate the crosscorrelation between the minute averaged velocities at the detectors D3 and D4 on the A1 (Fig. 13). If the platoons were stable the order of the vehicles passing a detector remains unchanged leading to a strong correlation of the velocity time-series. Vehicles on the left lane need about $30 \mathrm{~s}$ to drive from $D 3$ to $D 4$. As one can see, there is indeed a strong correlation of both sites at $\tau=1 \mathrm{~min}$, indicating that the lifetime of the platoons may have large 
values and moving structures can be identified even in free flow.

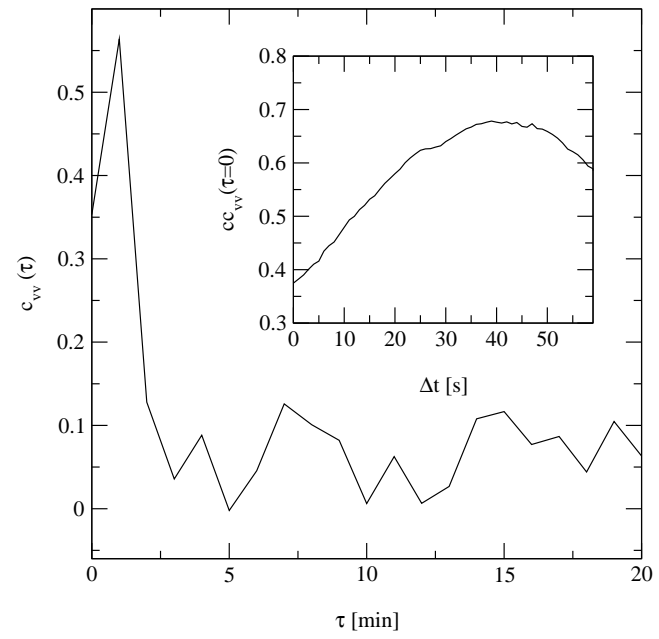

FIG. 13. Cross-correlation between the minute averaged velocities at the detectors D3 and D4 of the left lane on the A1 on the 08-10-2000. The inset shows the cross-correlation for $\tau=0$ of the velocity time-series at D4 and the by $\Delta$ seconds shifted velocity time-series at D3.

The analysis of single-vehicle data allows to specify the lag between the two time-series by varying the displacement of the two time-series continuously. The starting point of the averaging interval of the time-series at D3 is shifted by $\Delta$ seconds and then, the cross-correlation of the two signals is calculated. If the two time-series match, the cross-correlation would show large values at $\tau=0$. Hence, it is possible to locate the maximal correlation which corresponds roughly to the average travel time $\Delta_{\max }$ given by $1150 \mathrm{~m} / 150 \mathrm{~km} / \mathrm{h} \approx 28 \mathrm{~s}$ (see inset of Fig. 13).

As mentioned before, the third data set provides information about the signal the inductive loop measures from each car. We therefore tried to identify the moving structures of free flow traffic not only on the minute averaged scale but also on the single-vehicle scale. Unfortunately, the consideration and comparison of the vehicles electric signal at both detectors fails for two reasons. First, the electric signal differs only slightly from car to car. Second, even on the right lane the sequence of cars changes due to lane changes and overtaking maneuvers. As a consequence, the number of vehicles on one lane is not conserved so that even the cumulative number of vehicles passing a measurement section cannot be compared [20]. Therefore an explicit identification of a vehicle at both measurement locations was difficult and made the calculation of the travel-time distribution impossible.

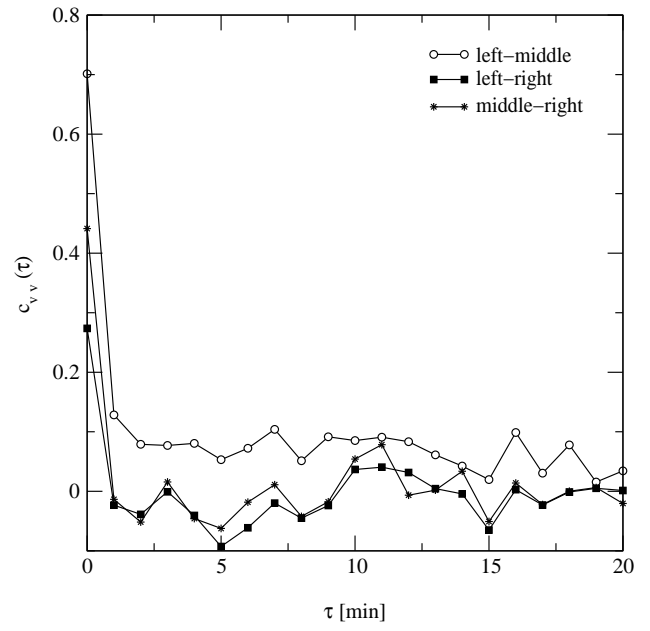

FIG. 14. Cross-correlation between the minute averaged velocities on the three lanes on the 04-28-2000 at detector D1 on the A3. Note, that the velocity time-series was detrended by a linear fit.

In [4] it was shown that in free flow traffic the correlation of the average velocity between different lanes is negligible. However, this result is contrasted by our analysis of the velocity time-series. In Fig. 14 the cross-correlation between the detrended average velocities on lane $i$ and lane $j$ is depicted. Obviously, there are strong correlations between the lanes, especially neighboring lanes are more correlated than the left with the right lane. Lane changes lead to a strong coupling between the lanes while the right lane is somehow decoupled from the other two due to the large amount of trucks. These lane changes are forced by the absence of a speed limit that may explain the differences to the results of [4] where a speed limit was in effect. As a result, daily fluctuations of the mean velocity and therefore of the flow are served first by the left and the middle lane while the flow of the right lane does not change significantly in the free flow regime.

\section{SYNCHRONIZED FLOW}

Synchronized traffic can be characterized by a large variance in flow and density measurements and a velocity that is considerably lower than in free flow traffic while the flow can show large values (Fig. 15). Although the minute averaged velocity is larger compared to a wide jam, the analysis of the single-vehicle data reveals the existence of vehicles with velocities smaller than $10 \mathrm{~km} / \mathrm{h}$, that is vehicles at rest were measured 21. 

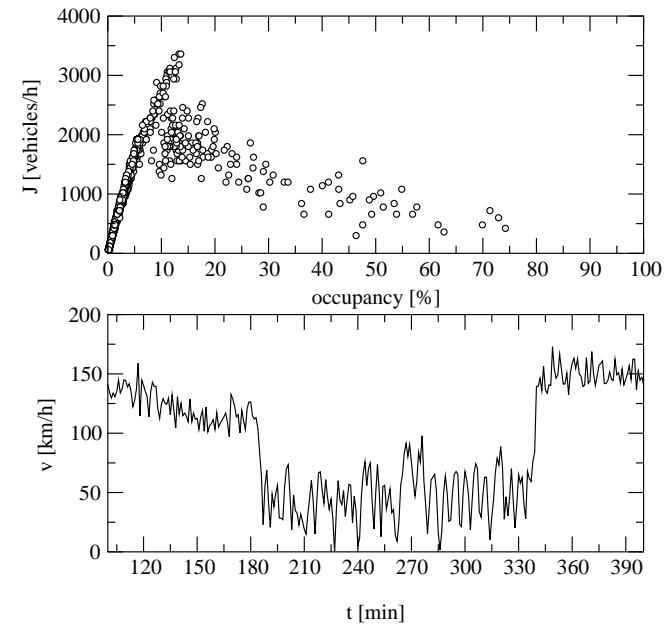

FIG. 15. Fundamental diagram and time-series of the velocity on the 05-11-2000 of the left lane at detector D1 on the A3.

In contrast to free flow, the time-headway distribution shows a non-trivial dependence on the density (Fig. 16). While the maximum of the distribution is shifted to larger values $(\approx 1.3 \mathrm{~s})$ and the variance is reduced, small time-headways do still exist. The probability of these small time-headways decreases with increasing density whereas the distribution in free flow can simply be rescaled by the density for small times. Note, that the distribution was calculated by the consideration of all days of the data set with synchronized traffic.

Compared to free flow traffic the length of clusters with small time-headways is decreased significantly (Fig. 17). The increased density in the synchronized regime is therefore responsible for the dissolution of the large platoons of the free flow regime. Moreover, because the velocity of the cars is now determined by the available space rather than by the desired velocity, the probability of cars piling up behind a slow car is drastically reduced. Now, safety reasons require larger distances to the preceding vehicle in order to prevent braking maneuvers. Note, that large clusters with time-headways larger than $2 \mathrm{~s}$ between the vehicles are obtained since the mean time-headway has the same order of magnitude.

Nevertheless, strong correlations of the velocity of the vehicles can be measured while the time-headway and the distance-headway are only weakly correlated [1. Thus, although the velocity of succeeding vehicles is strongly synchronized their gaps can vary considerably. As a result, platoons cannot be recognized.

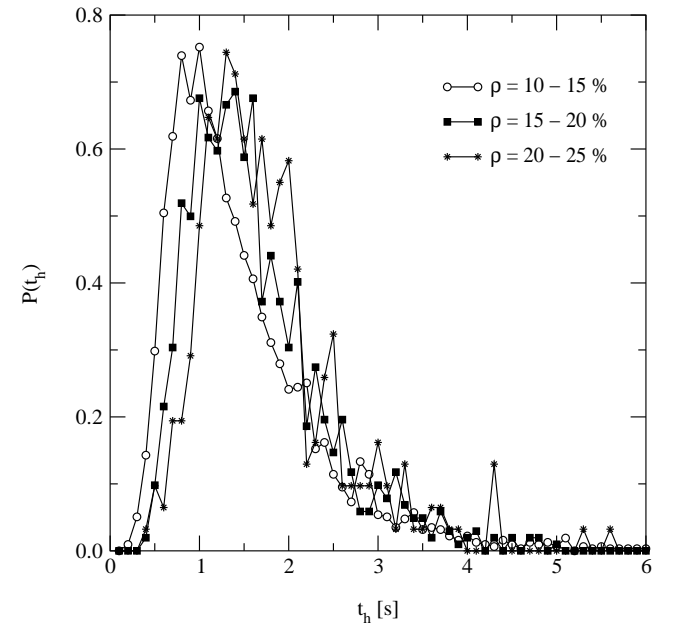

FIG. 16. Time-headway distribution in the synchronized regime for all days of the left lane at detector D1 on the A3.

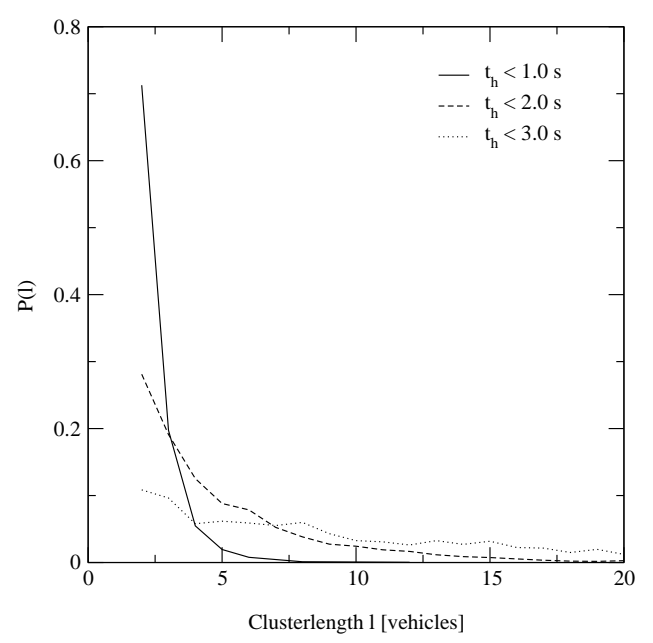

FIG. 17. Distribution of the cluster length on the left lane for different time-headways in the synchronized regime.

Also for the velocity distributions we found significant differences to free flow traffic. In synchronized traffic small velocities have, compared to a Gaussian distribution, a much higher weight (Fig. 18). The form of the velocity-distribution is probably a consequence of the accelerating and braking vehicles. Our result is of special importance for some macroscopic models of traffic flow [2], that assume Gaussian distributions of speeds, clearly incompatible with our empirical findings.

As shown in [4] the velocity-headway curve gives information about the average velocity of the vehicles at a given headway. 


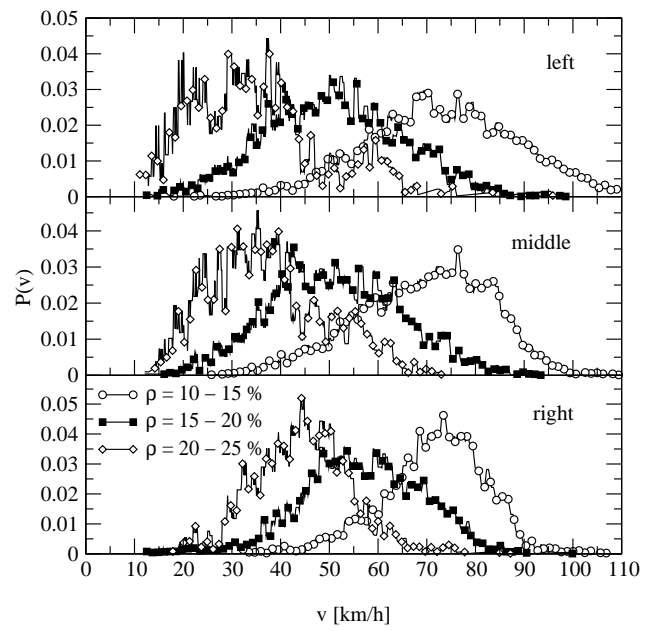

FIG. 18. Corrected distribution of the velocity in the synchronized regime.

In the free flow regime large velocities are reached even for very small distances but in synchronized traffic the asymptotic velocity is reduced considerably (Fig. 7). Again, the velocity-headway curve was obtained by averaging over all days with synchronized traffic. Since the vehicles drive slower than the gap allows the gap usage is only suboptimal. Moreover, the velocity of a vehicle at a given distance depends strongly on the traffic density. The larger the density the lower the velocity which allows the driver to anticipate breakdowns.

The transition from free flow to synchronized traffic is characterized by the synchronization of the speed on different lanes and a sharp drop of the velocity. This synchronization is a result of lane changes in order to align the density of the lanes [3, 25] rather than a consequence of the difference between the maximum velocities of the vehicles.

In Fig. 19 the time-series of the velocity measurements for the three lanes is shown. A slow decrease of the average velocity on the left and the middle lane can be seen until a sharp drop of the velocity occurs. Obviously, the breakdown happens at the same time for all three lanes. In contrast to the velocity, the density difference between the lanes decreases continuously and completely vanishes at the transition (Fig. 20). The synchronization of the lanes therefore happens continuously.

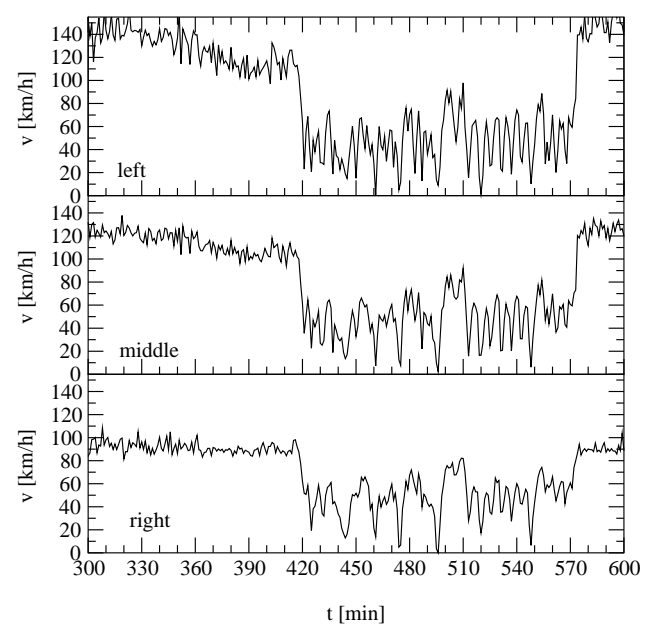

FIG. 19. Velocity time-series at detector D1 on the A3 on the 05-11-2000 for all three lanes.

The calculation of the cross-correlation between the velocities, the densities and the flows on the three lanes reveals that the lanes are strongly correlated, e.g., synchronized. As a consequence of this synchronization the distribution of the velocity, the distance-headway and the time-headway are nearly the same for each lane. Thus the basic driving behavior in synchronized traffic is determined neither by the density nor by the lane but depends only on the traffic state.

Again, the cross-correlation analysis of the velocity time-series between the detectors D3 and D4 on the A1 allows the identification of moving structures (Fig. 21). The difference in the life-time of the synchronized states at each location suggests the existence of a bottleneck far upstream of D4 where a transition from free flow to synchronized traffic has occurred. 


\section{WIDE MOVING JAMS}

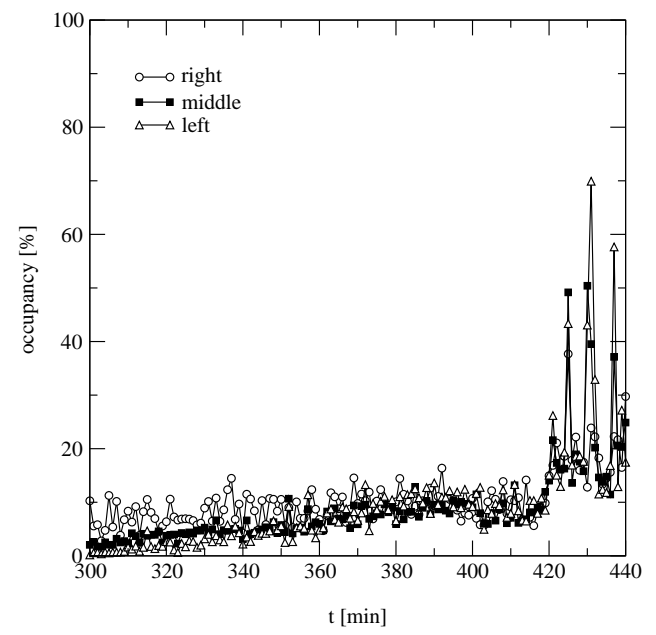

FIG. 20. Occupancy time-series of the three lanes at detector D1 on the A3 on the 05-11-2000.
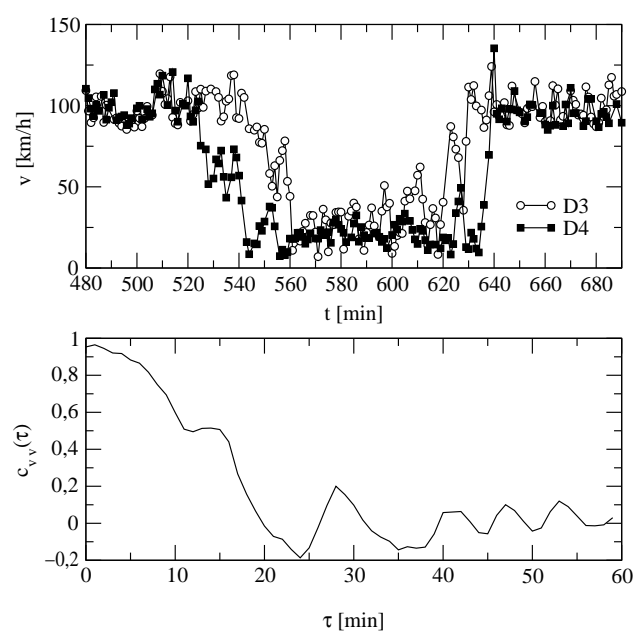

FIG. 21. Cross-correlation (bottom) between the velocity time-series (top) of synchronized traffic of the middle lane on the 02-20-2001 at the detectors D3 and D4 on the A1.

As an indication for the stochasticity of the jam formation mechanism velocity fluctuations increase with the distance to the bottleneck, finally leading to a pinch region with small jams. The propagation of these small jams leads to a small decay of the cross-correlations of the order of magnitude of $10 \mathrm{~min}$ which is about the period of the jam emergence at one measurement location. However, the upstream velocity of these small jams cannot be determined due to their continuous excitation and extinction.
In contrast to free flow and synchronized traffic wide moving jams are moving structures that are bounded by two fronts where the vehicle speed changes considerably. The flow and the velocity inside the wide jam are negligible whereas the density has large values (Fig. 23). These large values of the density can be traced back to a large number of vehicles that passed the detector rather than to a single car at rest which occupies the detector for a long time. Shifting the time for $60 \mathrm{~s}$ the minute averaging starts and averaging over these values in order to obtain a mean density shows no decrement of the density.

In the fundamental diagram the wide jam can be identified by a triangle structure at large densities and small flows (Fig. 22). Two effects have to be taken into account in the interpretation of the data for wide jams: 1) Wide jams are not compact in the sense that cars are piled up bumper-to-bumper, but there can still exist relatively large gaps inside. These gaps lead to small values of the density and the flow. 2) Standing vehicles can block the detector for a certain time. This results in large densities but small flows.

The characteristics of a wide jam are the upstream moving velocity and the outflow from the jam if free flow is formed in the outflow. As one can see in Fig. 22 the transition from a wide jam to free flow is accompanied by a jump of the velocity to values lower than in free flow traffic while the density and the flow are considerably larger (Fig. 23) determining $J_{\text {out }}$ and $\rho_{\text {out }}$ [3] to be 2200 vehicles $/ \mathrm{h}$ and $10 \%$. In addition, the difference of the outflow from a jam to free flow traffic decreases from the left to the right lane due to the decreasing average velocity.
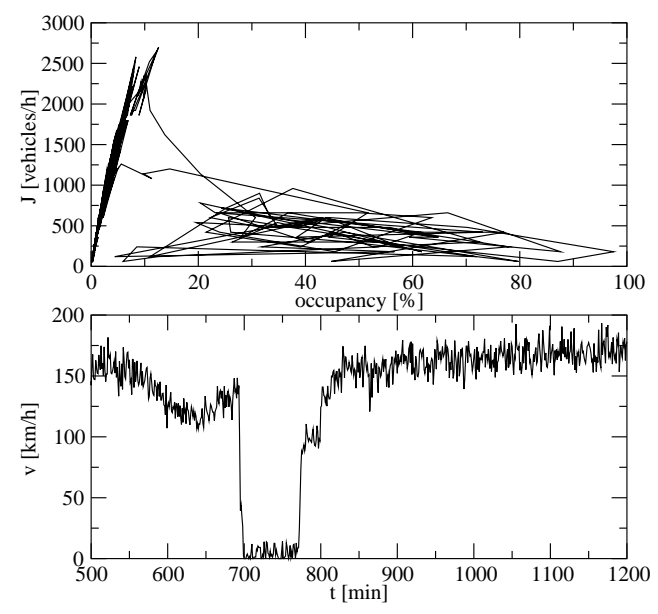

FIG. 22. Fundamental diagram and velocity time-series of the left lane on the A3 at detector D2 on the 04-15-2000. 


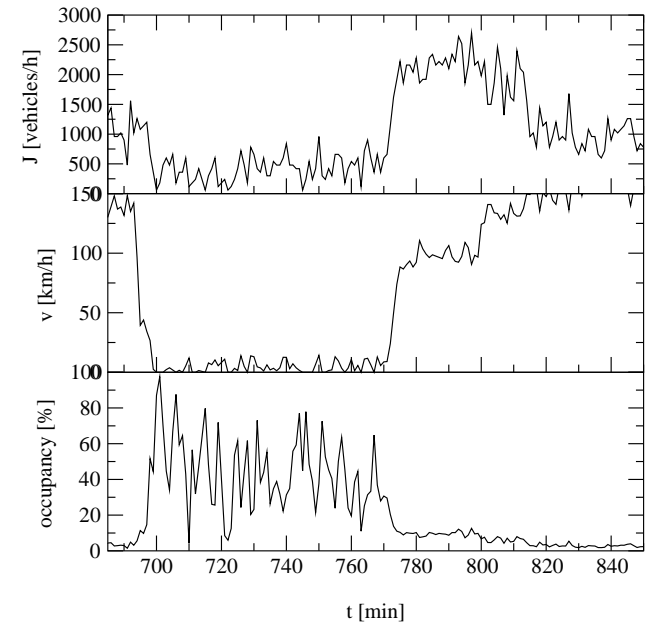

FIG. 23. Time-series of the flow, the velocity and the occupancy of the left lane on the A3 at detector D2 on the 04-15-2000.

Inside the jam the velocity and the flow are negligible. Since vehicles can only move due to the propagation of holes, the velocity inside a wide jam is distributed only in a very small range (Fig. 24).

Note, that the cut-off is due to measurement restrictions, e.g., velocities smaller than $10 \mathrm{~km} / \mathrm{h}$ are not detected. As mentioned before, the calculation of the headways is only correct under the assumption that the velocities of two consecutive vehicles do not change significantly. However, relatively large values of the time and the distance headway become visible (Fig. 24) which can be explained by gaps within the jam. Hence, the wide jam is not compact but gaps allow the movement of the vehicles.

If vehicles with a velocity smaller than $10 \mathrm{~km} / \mathrm{h}$ pass the detector the velocity is not detected but instead of the length of the vehicle its time blocking the detector is given. This time, the occupancy time $\Delta t$, allows to estimate the time, a car at rest needs to accelerate 椆. In Fig. 25 the distribution of the occupancy time is shown. Obviously, a vehicle needs a minimal time of about 2 $\mathrm{s}$ to accelerate. As a characteristic property of a wide jam [11] this time determines (including the density inside the jam) the escape rate from a jam and thus its outflow. As a consequence of the gaps inside the jam a stop-and-go pattern forms that leads to an uncorrelated movement of the vehicles. However, in contrast to the autocorrelation function of the time-headway and

\footnotetext{
${ }^{\ddagger}$ Since it is possible that the vehicle has to stop at the detector the assumption of a constant velocity is no longer valid. Thus, the calculation of the time-headway is not possible.
}

the distance-headway the correlation of the single-vehicle velocity decays slower (Fig. 26).
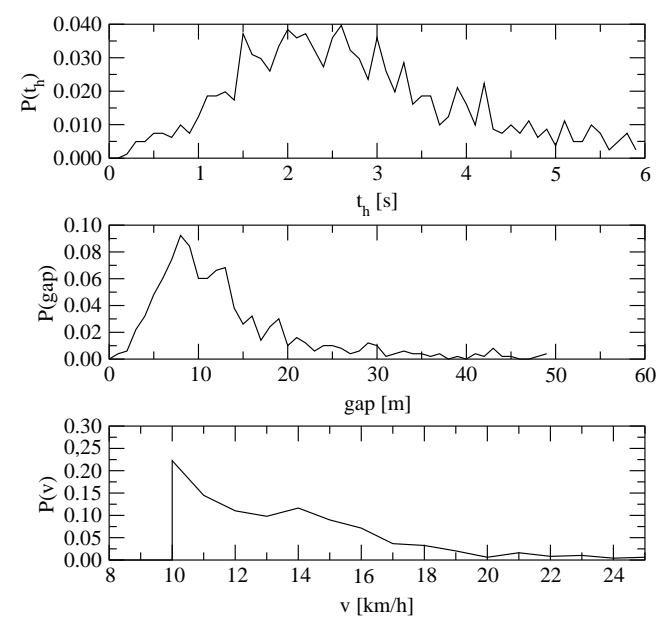

FIG. 24. Distribution of the time and the distance headway and the velocity in a wide jam for the left lane on the A3 at detector D2 on the $04-15-2000$ and at detector D1 on the 05-02-2000.

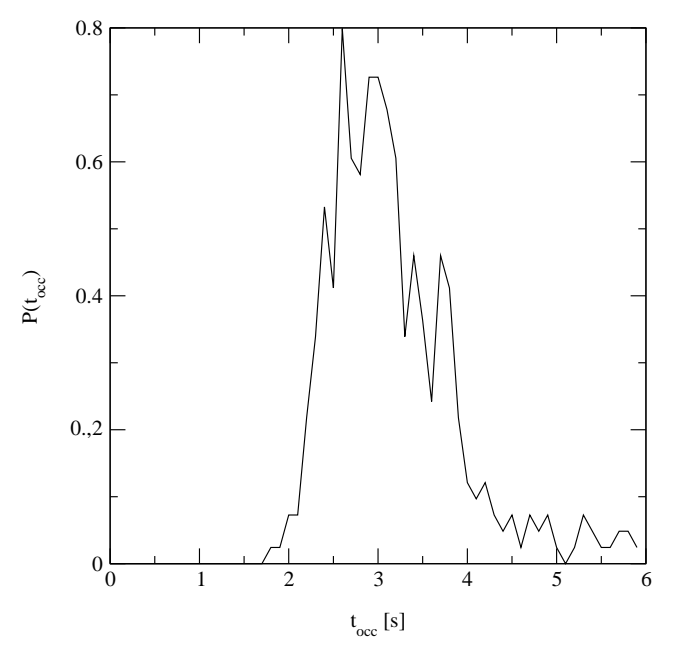

FIG. 25. Distribution of the time the detector is occupied by a car with a velocity smaller than $10 \mathrm{~km} / \mathrm{h}$ in a wide jam.

Thus, weak correlations between the vehicles on very short length scales exist that can be traced back to the successive acceleration of stopped vehicles.

In analogy to synchronized traffic the transition from free flow traffic to wide jams is accompanied by an alignment of the densities on the different lanes. In contrast to the transition from free flow to synchronized traffic the 
velocity difference between the lanes does not decrease indicating that the breakdown happens due to a large perturbation. Nevertheless, the breakdown of the velocity time-series can be observed at the same time on all lanes which is a consequence of the synchronization of the lanes.

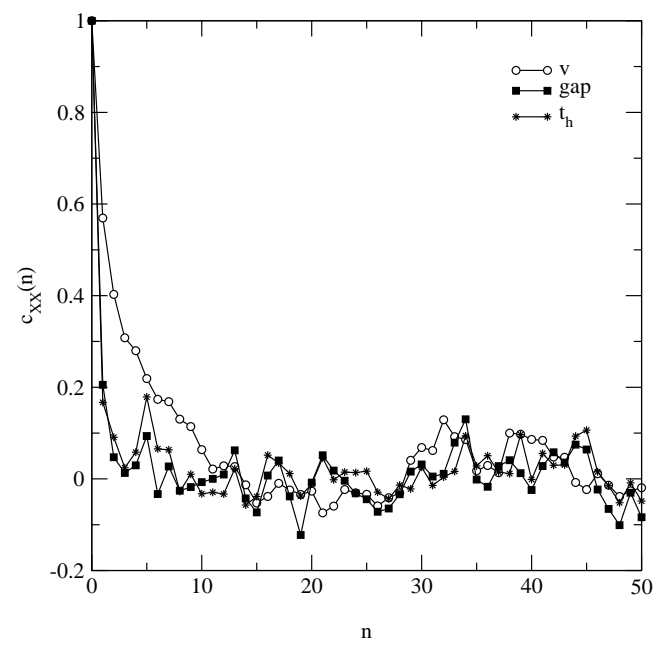

FIG. 26. Autocorrelation of the single-vehicle data of the velocity and the time and the distance-headway in a wide jam on the left lane on the 04-15-2000 at the detector D2 on the A3.

As the next step we analyze the so-called lane-usage inversion. The lane-usage inversion is a special phenomenon observed on German highways [22 and concerns the distribution of the total flow on the lanes. For low densities nearly all the flow can be measured on the right lane.

However, above a certain density, the inversion point, most of the flow can be found on the left lane rather than on the right lane. This special observation can be explained by the legal restrictions of highway traffic in Germany. Since the left lane is the designated fast lane, vehicles must drive on the right lane where overtaking is forbidden. As a consequence, at larger densities cars keep on moving on the left lane in order to avoid being trapped behind a slow car on the right lane. Thus, more and more flow can be found on the left rather than the right lane. This behavior is contrasted by observations on American freeways [23, 24].

In Fig. 28 the distribution of the flow on the three lanes averaged for the whole data set of the A3 is depicted. In contrast to observations of two-lane traffic, in three-lane traffic three inversion points are visible. At a flow of about 1000 vehicles/h the weight of the distribution is shifted from the right to the middle lane. At a total flow of about 2500 vehicles/h the second inversion point is reached and more flow can be found on the left than on the right lane. Finally, at flows larger than 3500 vehicles/h the share of the flow is larger on the left than even on the middle lane.

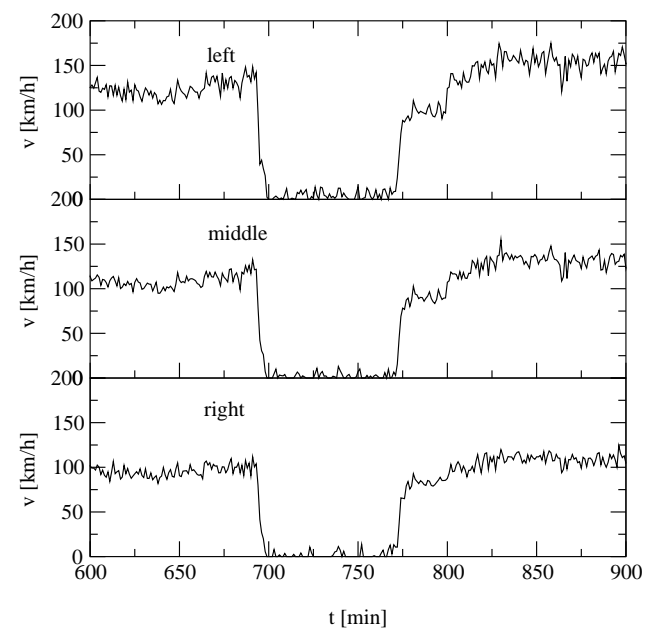

FIG. 27. Time-series of the velocity for the three lanes on the 04-15-2000 at detector D2 on the A3.

In contrast to the flow distribution the distribution of the total occupancy shows no inversion points. As mentioned before, the transitions from free flow to either synchronized traffic or wide jams is accompanied by a alignment of the density as shown in Fig. 29. Right before the transition the density is evenly distributed on the three lanes.

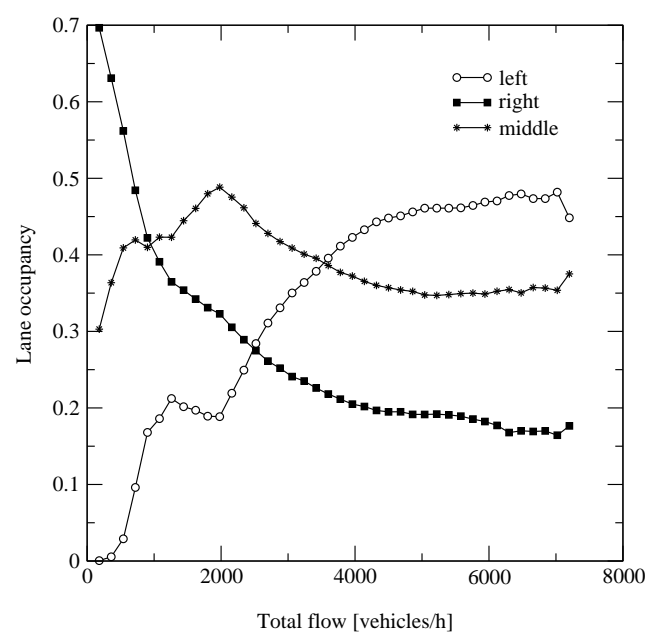

FIG. 28. Distribution of the total flow on the three lanes. 


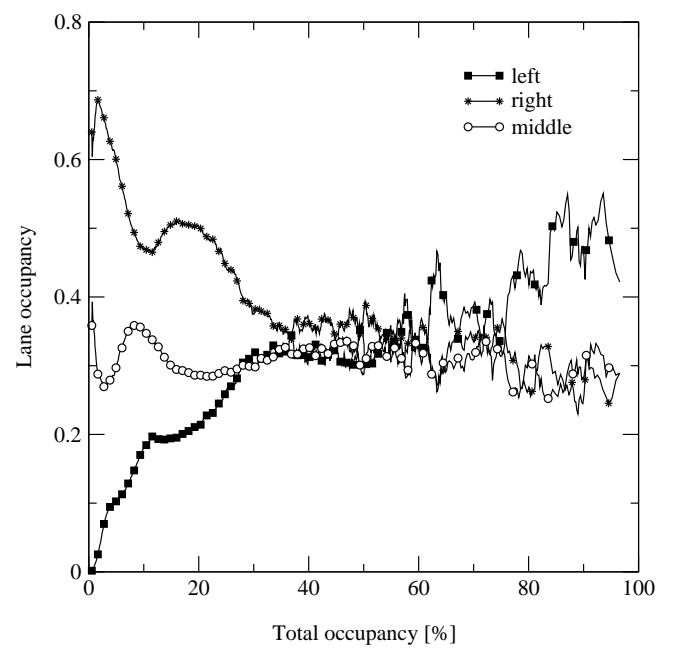

FIG. 29. Distribution of the total occupancy on the three lanes. The data were smoothed by a running average.

For all densities there is a negative density gradient from the right to the left lane. With increasing density the difference between the lanes decreases but for no density more vehicles are on the left than on the right lane. However, as mentioned in section II occupancy gives the percentage of the measurement time the detector is covered by vehicles. Therefore, the slower the vehicles are the larger the occupancy since slow vehicles occupy the detector a longer time than fast vehicles. In order to estimate the spatial density it is necessary to measure the mean length of the vehicles on the individual lanes. With the values $L_{\text {left }}=4.25 \mathrm{~m}, L_{\text {middle }}=4.57 \mathrm{~m}$ and $L_{\text {right }}=8.3 \mathrm{~m}$ the density can be calculated via eqn.(2).

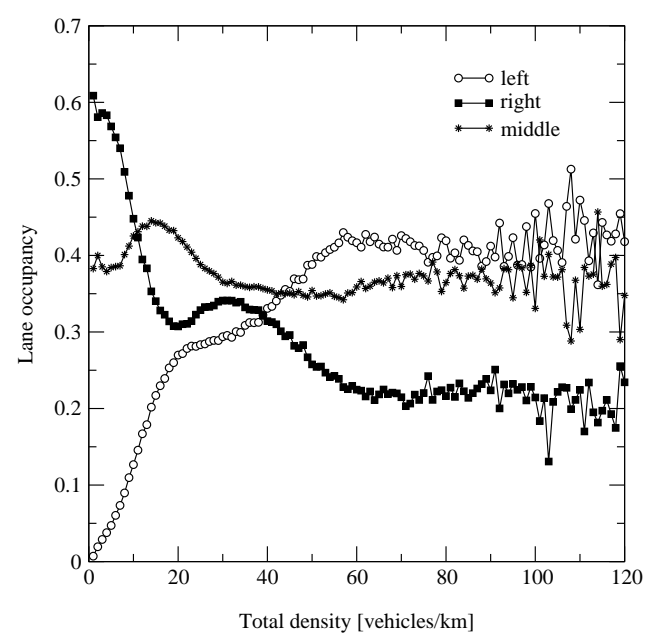

FIG. 30. Distribution of the total spatial density on the three lanes.
As one can see in Fig. 30 there is indeed a lane-usage inversion. At densities well below a certain point more vehicles are on the left than on the right and the middle lane, leading to a negative flow gradient from left to right. In addition, the cross-over of the density distribution occurs analogously to the flow distribution at three inversion points. As a consequence of the large amount of trucks on the right lane, the left and the middle lane show nearly the same lane occupancy which is considerably larger compared to the right lane. This fact is another indication for the decoupling of the right from the left and the middle lane.

Thus, the lane usage inversion can be traced back on the one hand to differences of the average velocity between the lanes that lead to larger flows from right to left and on the other hand to an asymmetric distribution of the vehicles on the lanes (the flow inversion can also be observed on highways with speed limit).

\section{CONCLUSION}

Our analysis of single-vehicle traffic data leads to a more elaborated microscopic picture of highway traffic. In the free flow state correlations between the vehicles are only visible on scales smaller than one minute. The correlations result from platoons, i.e., clusters of cars driving bumper-to-bumper with very small time-headways. Within these clusters the vehicles are driving with nearly the same velocity leading to a large temporal stability of the platoons. With increasing size of the platoons we observe a larger distance between the vehicles inside the platoon.

In contrast to highways without speed limit, with speed limit drivers tend to drive with larger temporal distance, i.e., the probability of very small headways is reduced considerably and the maximum of the timeheadway distribution is shifted towards larger values. This may indicate that speed-limits lead to a reduction of accidents. But even more efficient than that would be the reduction of the number of drivers driving with time-headways comparable to their reaction time, e.g., by equipping cars with devices that adjust automatically the distance to the leading car.

Another remarkable effect that can already be observed in the free flow regime is the synchronization of speeds on the different lanes, which was predicted by different simulation studies [25].

The velocity distributions, that are important as an input for macroscopic traffic models [2], have the expected Gaussian shape for densities of $5-10 \%$. Surprisingly this is not true for very low densities. There the distribution functions show a slight asymmetry, with an increased weight of large speeds.

The synchronized traffic state is known to be characterized by increased fluctuations, e.g., of the velocity time 
series, compared to free flow traffic. On a microscopic level these fluctuations influence the time-headway distributions on short time scales. The short headways are systematically suppressed and the maximum of the distributions is shifted towards larger headways. This effect may be explained by the tendency of drivers to avoid frequent accelerations and brakings [26]. Other consequences of the larger velocity fluctuations are the more rapid dissolution of the platoons in synchronized traffic and the much higher weight of small velocities. The low speed part of the velocity distribution is probably governed by acceleration and braking vehicles.

Wide jams can be identified by a triangular shape in the fundamental diagram. Since they are not compact the transportation of holes leads to small flows at small densities. The analysis of the time a vehicle at rest covers a detector allows to estimate its acceleration time. This time determines the outflow from a jam and is characteristic for wide jams. Our result of about $2 \mathrm{~s}$ confirms the value found in 11 .

The transition from free flow to either synchronized traffic or wide moving jams is accompanied by a continuous alignment of the density on the different lanes. The breakdown of the velocity happens simultaneously which is a consequence of the synchronization of the vehicle's speed on the lanes.

Empirical studies reveal that on German two-lane highways the flow is distributed asymmetrically on the lanes. This lane usage inversion is a result of the asymmetric distribution of the vehicles on the lanes and can be found even on three-lane highways. It is increased by a negative velocity gradient from the left to the right lane on highways without speed limit.

Our analysis confirms the basic results of former empirical studies $\llbracket, 5$. In particular, the characteristics of the time-headway distribution and the velocity-distance curve have been found in both data sets. The new data allowed to clarify an open question about the occurrence of peaks in the time-headway distribution [4] that are most likely an artifact of the internal data handling of the inductive loops. The present results show that a peak at a headway of $1.8 \mathrm{~s}$ does not exist.

Finally, our results confirm that the basic driving strategies implemented in a recent cellular automaton traffic model [26] match quite well the empirical situation. Of special importance are anticipation effects, but also the reduced gap acceptance in synchronized traffic. We also want to stress the fact that the different traffic states, which have been identified by means of a spatiotemporal analysis of time-averaged data, differ equally well with respect to their microscopic structure. ¿From our point of view, this coherence between microscopic and macroscopic empirical analyses supports the validity of the classification scheme.

Acknowledgments: The authors are grateful to the Landesbetrieb Straßenbau NRW (especially Mr. Thomas) for the data support and to the Ministry of Economics and Midsize Businesses, Technology and Transport for the financial support. We thank Mr. Wefer from BIANDI Informationssysteme for useful discussions and his technical advice concerning the inductive loops. L. S. acknowledges support from the Deutsche Forschungsgemeinschaft under Grant No. SA864/1-2.

\section{APPENDIX A:}

The main problem in the analysis of empirical timeseries are irregular observation times or gaps in the observations, i.e., data which are no evenly spaced. In contrast, the classical definition of the correlation function of two observables $X$ and $Y$ measured at $N$ points

$$
c_{X Y}(\tau)=\frac{1}{N} \sum_{n=1}^{N-\tau} X_{n} Y_{n+\tau}
$$

requires evenly spaced data. There are some approaches for filling the gaps in the time-series, but nevertheless the original time-series is changed by these methods. In order to circumvent these problems, the correlation is calculated in the frequency domain and then transformated back to the time domain. The correlation theorem 27] states, that the cross spectrum of two functions is the Fourier transform $F$ of its cross-correlation function:

$$
c_{X Y}=F^{-1}\left[F_{X}(\omega) F_{Y}^{*}(\omega)\right] .
$$

The Fourier transform of unevenly spaced data $\left\{X_{n}=\right.$ $\left.X\left(T_{n}\right), n=1,2, \ldots, N\right\}$ is computed after [28] and is defined to be:

$$
F_{X}(\omega)=F_{0} \sum\left(A X_{n} \cos \left(\omega t_{n}^{\prime}\right)+i B X_{n} \sin \left(\omega t_{n}^{\prime}\right)\right)
$$

where

$$
F_{0}(\omega)=\sqrt{\frac{N}{2}} e^{-i \omega t_{1}}, \quad t_{n}^{\prime}=t_{n}-\tau(\omega)
$$

and

$$
A(\omega)=\frac{1}{\sqrt{\sum_{n} \cos ^{2}\left(\omega t_{n}^{\prime}\right)}}, \quad B(\omega)=\frac{1}{\sqrt{\sum_{n} \sin ^{2}\left(\omega t_{n}^{\prime}\right)}}
$$

and

$$
\tau(\omega)=\frac{1}{2 \omega} \tan ^{-1}\left(\frac{\sum_{n} \sin \left(2 \omega t_{n}\right)}{\sum_{n} \cos \left(2 \omega t_{n}\right)}\right) .
$$

$t_{1}$ is determined by the origin of time. See [28] for details of the computing the Fourier transform and for a Fortran code. Inversion back to the time domain can be accomplished using the Fourier transform for evenly spaced frequencies. 
[1] D. Chowdhury, L. Santen, A. Schadschneider, Physics Reports 329, 199 (2000).

[2] D. Helbing, Rev. Mod. Phys. 73, 1067 (2001).

[3] B. S. Kerner, Networks and Spatial Economics, 1, 35 (2001).

[4] L. Neubert, L. Santen, A. Schadschneider, M. Schreckenberg, Phys. Rev. E 60, 6480 (1999).

[5] B. Tilch, D. Helbing, In: Traffic and Granular Flow '99, p. 333, D. Helbing, H. J. Herrmann, M. Schreckenberg, D. E. Wolf (eds.) Springer (Heidelberg, 2000).

[6] D. Helbing, A. Hennecke, M. Treiber, Phys. Rev. Lett. 82, 4360 (1999).

[7] H. Y. Lee, H. W. Lee, D. Kim, Phys. Rev. E 62, 4737 (2000).

[8] M. Treiber, A. Hennecke, D. Helbing, Phys. Rev. E 62, 1805 (2000).

[9] B. S. Kerner, H. Rehborn, Phys. Rev. E 53, R4275 (1996).

[10] M. Koshi, M. Iwasaki, I. Ohkura, in Proceedings of the 8th International Symposium on Transportation and Traffic Theory, edited V. F. Hurdle, E. Hauer, G. N. Stewart, (University of Toronto Press, Toronto, Ontario,1983), p. 403.

[11] B. S. Kerner, Phys. Rev. Lett. 81, 3797 (1998).

[12] B. S. Kerner, H. Rehborn, Phys. Rev. E 53, R1297 (1996).

[13] G. Diedrich, L. Santen, A. Schadschneider, J. Zittartz, Int. J. of Mod. Phys. C 11, 335 (2000).

[14] W. Knospe, L. Santen, A. Schadschneider, M. Schreckenberg, Phys. Rev. E 65, 015101 (2002).

[15] V. Popkov, G. M. Schuetz, Europhys. Lett. 48, 257 (1999).

[16] G. M. Schuetz: Exactly Solvable Models for Many-Body Systems Far From Equilibrium, in: C. Domb, J. L. Lebowitz (eds.): Phase Transitions and critical phenomena, vol. 19, 1, Academic Press (2001).

[17] D. Helbing, Phys. Rev. E 55 (3), 3735 (1997).

[18] A. D. May, Traffic flow fundamentals (Prentice Hall, Englewood Cliffs, 1990).

[19] M. N. Islam, P. C. Consul, Transpn. Res. B 25 (5), 365 (1991).

[20] M. J. Cassidy, J. R. Windover, Transpn. Res. A 32 (2), 129 (1998).

[21] B. S. Kerner, Physics World 8, 25 (1999).

[22] U. Sparmann, In: Forschung Straßenbau und Straßenverkehrstechnik Heft 263, eds: Bundesminister für Verkehr, 1978 (Bonn-Bad Godesberg).

[23] Fred L. Hall, Tenny N. Lam, Transp. Res. A 22, 45 (1988).

[24] Gang-Len Chang, Yang-Ming Kao, Transp. Res. A 25, 375 (1991).

[25] W. Knospe, L. Santen, A. Schadschneider, M. Schreckenberg, Physica A 265, 614 (1999); D. Chowdhury, D. E. Wolf, M. Schreckenberg, Physica A 235, 417 (1997).

[26] W. Knospe, L. Santen, A. Schadschneider, M. Schreckenberg, J. Phys. A 33, L477 (2000).

[27] G. M. Jenkins, D. G. Watts, Spectral Analysis and Its Applications (Holden-Day, San Francisco, 1968).

[28] J. D. Scargle, Ap. J. 343, 874 (1989). 